\title{
La queue qui remue le chien
}

Métamorphose de la finance due à son informatisation

The Tail that wags the dog. Metarmophosis of finance due to data-processing

\section{Paul Jorion}

\section{(2) OpenEdition}

\section{Journals}

Édition électronique

URL : https://journals.openedition.org/tc/5028

DOI : $10.4000 /$ tc. 5028

ISSN : 1952-420X

Éditeur

Éditions de l'EHESS

Édition imprimée

Date de publication : 30 juin 2010

Pagination : 671-685

ISSN : 0248-6016

Référence électronique

Paul Jorion, «La queue qui remue le chien », Techniques \& Culture [En ligne], 54-55 | 2010, mis en ligne le 30 juin 2013, consulté le 29 septembre 2022. URL : http://journals.openedition.org/tc/5028 ; DOI : https://doi.org/10.4000/tc.5028 


\section{LA QUEUE QUI REMUE LE CHIEN}

\section{Métamorphose de la finance due à son informatisation}

in Techniques et culture 23-24, 1994: 307-349

Nombreux sont les domaines de l'activité humaine qui ont su tirer parti de la deuxième vague d'informatisation, celle liée à l'invention de l'ordinateur personnel puis de la station de travail fonctionnant sous système d'exploitation Unix. Mais rares sans doute sont ceux qui ont su exploiter cette nouvelle technologie de manière aussi exhaustive que ne l'a fait la finance. Une des raisons en est qu'une évaluation du bénéfice procuré par l'informatisation se fait plus aisément qu'ailleurs en finance et que l'investissement y a posé peu de problèmes, la liquidité y ayant été, jusqu'à très récemment, disponible à satiété.

La technologisation de la finance s'est opérée partout de la même manière; son coût a entraîné l'élimination des moins riches, ne laissant en présence sur le plan mondial que les institutions les mieux équipées sur le plan technique. Cette évolution a contribué à l'unification du domaine financier à l'échelle de la planète, et n'est en fait que l'une des manifestations de la globalisation de l'économie qui a caractérisé les vingt dernières années.

John Maynard Keynes exécrait la relation qui connectait l'économie de la production à l'« activité d'un casino », expression qu'il utilisait pour caractériser l'activité financière proprement dite (in Skidelsky 1992 : xxviII). Or, les revenus générés par la finance sont de plus en plus aléatoires, soumis à de grandes variations difficilement maîtrisables, et ceci en raison même de l'informatisation. La rapidité dans la transmission de l'information a favorisé les influences réciproques entre marchés, rendant plus probables les effets d'emballement sur les prix (ou sur les taux). La réduction des marges de profit d'institutions soumises à une concurrence de plus en plus serrée, a encouragé ces institutions à tenter de se distinguer vis-à-vis du client par des activités de plus en plus spéculatives, autrement dit, de plus en plus risquées. Du coup, l'édifice de la finance est devenu plus 
solidaire et chacune de ses parties s'est vue exposée aux risques pris par chacune des autres. Et comme les enjeux sont ici les prix des matières premières, le financement des sociétés et le niveau des taux d'intérêt, les ondes de choc des crises financières se propagent désormais toujours plus loin et plus longtemps dans l'économie productive tout entière.

Le phénomène est à première vue d'ordre plus social que culturel. Mais il a trouvé sa voie vers les représentations, et le corps social se conçoit aujourd'hui comme serti de manière naturelle au sein d'un contexte économique intrinsèquement instable. Le choc pétrolier de 1973 a modifié les consciences, sinon une fois pour toutes, du moins sur la vingtaine d'années qui nous en séparent, et la culture a entériné un renversement dans la conception du «normal », de l'immuable vers le chaos. La cyclicité des crises, qui n'était jusqu'à la fin des années soixante qu'un thème de propagande marxiste, s'est imposée comme paradigme: on ne conçoit plus dorénavant la crise économique comme un événement monstrueux dont la fin est proche dans l'histoire humaine mais comme une fatalité inéluctable, la stabilité économique n'étant que cette plage heureuse séparant deux crises dont le retour cyclique est à la fois certain et imprévisible, et que seuls les « atterrissages en douceur » planifiés par les Banques Centrales permettraient d'amortir.

\section{Contexte technique de la finance}

Les organismes procédant à des opérations sur instruments financiers ont généralement une structure feuilletée à trois niveaux au moins: le Front-Office ou « salle de marchés » où des $c$ prennent des positions sur les marchés (achats, ventes de titres ou d'obligations, prêts, emprunts, opérations au comptant ou à terme sur des obligations, des matières premières, des devises, options sur tous les produits précédemment nommés, etc.); un Back-Office qui procède à l'enregistrement, à la confirmation et au suivi des opérations du Front-Office (reporting); et un Middle-Office (d'introduction plus récente) qui produit des états synthétiques des positions en vue de la gestion du risque sous toutes ses formes - risque dû essentiellement aux variations imprévisibles des taux d'intérêt, aux variations des cours de change entre devises et risque de crédit découlant de l'insolvabilité éventuelle des contreparties aux positions prises. La Comptabilité de l'établissement et son Contrôle de Gestion veillent à la présentation de résultats aux actionnaires, au fisc et aux autorités de tutelle des activités financières (Commission Bancaire).

Loutil de travail du trader de Front-Office est un complexe constitué de lignes téléphoniques le connectant à des courtiers ou à la Bourse, d'écrans affichant des cotes et des prix, d'un tableur ou feuille de calcul et d'un ensemble de logiciels spécifiques servant par exemple à la cotation des produits financiers complexes qu'il ou elle est susceptible d'acheter ou de vendre, d'emprunter ou de prêter, tels que swaps ou options (logiciels généralement développés par l'informatique « maison »). Les systèmes d'enregistrement de l'employé de Back-Office sont en général de vastes progiciels de gestion et de traitement de bases de données loués à des maisons spécialisées qui en assurent la maintenance. L'extension des télécommunications a permis l'affichage des nouvelles et des cotations en temps réel sur écran, le trading direct (passage d'ordres de vente et d'achat) par ordinateur (à l'aide d'un modem connectant l'ordinateur à une ligne téléphonique) et l'échange de correspondance contractuelle et de confirmation par fax. 
Ces développements techniques, par informatisation et par extension des télécommunications, ont affecté la finance internationale de deux manières, l'une commune à de nombreux autres domaines de l'activité humaine où ils sont intervenus de la même façon, l'autre spécifique à la finance. De ce dernier point de vue, celle-ci a subi au cours des dix dernières années une transformation majeure.

\section{Effets classiques de l'informatisation sur la finance}

L'influence de l'informatisation commune à la finance et à d'autres domaines consiste dans le remplacement de l'homme par la machine pour les tâches les plus répétitives et les plus aisément susceptibles d'être automatisées, à savoir partout où s'exercent des activités purement administratives ou comptables. On a assisté ainsi aux cours des cinq dernières années à des réductions massives d'effectifs dans les domaines bancaire et boursier. Mais davantage que quantitative, la transformation des personnels a été qualitative: les traders et les personnels impliqués dans la recherche et le développement ont de plus en plus souvent été recrutés parmi les spécialistes les plus qualifiés en mathématiques appliquées: ingénieurs, informaticiens de haut niveau, voire physiciens ${ }^{1}$.

La manifestation la plus visible des changements quantitatifs dans la structure des équipes s'observe dans la variation de proportion des personnels affectés à diverses fonctions selon le degré d'informatisation possible de leurs tâches. Le rapport en nombre des personnels de Front-Office - se consacrant donc aux opérations financières proprement dites - et de Back-Office - se consacrant aux tâches d'enregistrement et de confirmation - s'est sensiblement modifié au cours des années récentes: le personnel de Back-Office a été considérablement réduit quand il n’a pas tout simplement disparu, les tâches de Back-Office étant alors entièrement sous-traitées par des organismes extérieurs, alors que le personnel de Front-Office restait stable en nombre et voyait, comme nous l'avons dit, sa qualification augmenter régulièrement.

\section{Spécificités de l'informatisation de la finance}

Cette manifestation du remplacement de l'être humain par la machine ne présente cependant aucun caractère propre au domaine financier et nous n'en dirons pas davantage ici. Ce qui nous retiendra en revanche, c'est la spécificité des implications de l'informatisation sur la finance, lesquelles se sont traduites par une modification dans la formation du prix des actions cotées en bourse, des taux d'intérêt et des cours de change.

Si l'on distingue la partie de l'économie fondée sur la production de celle fondée sur une simple interaction de la monnaie avec elle-même - ce qui fera office ici de définition pour la finance ${ }^{2}-$, on constate que l'informatisation a conduit à subordonner toujours davantage l'économie de la production à l'économie de la monnaie. Selon une image qui fit florès au cours des mois de février et mars 1994, c'est désormais la « queue » de la finance qui fait bouger le « chien » de l'économie (voir par exemple les titres de deux 
articles publiés, l'un en Grande-Bretagne, When the tail wags the market dog, de Rupert Bruce, février 1994, l'autre, aux États-Unis de Jose De La Torre, International finance has become the dog-wagging tail of the '90s, mars 1994).

Dans un ouvrage récent, Fabozzi, Modigliani (prix Nobel d'économie) et Ferri font observer:

« Un grand nombre d'instruments financiers requièrent l'usage d'ordinateurs pour les créer initialement aussi bien que pour en superviser le fonctionnement ensuite. La mise en œuvre des stratégies de trading utilisant ces instruments financiers requiert elle aussi des ordinateurs ainsi que des réseaux de télécommunication. En l'absence de certains progrès dans les technologies informatique et de télécommunication, certaines innovations financières auraient été impossibles. Bien que les instruments financiers et les stratégies de trading créés par certains acteurs présents sur les marchés puissent être trop complexes pour être utilisés par certains autres, le degré de sophistication des acteurs, tout particulièrement en termes de formation mathématique, s'est accru, permettant l'intégration de certains instruments et stratégies de trading complexes. » (1994 : 29).

Plusieurs implications de l'informatisation de la finance sont mentionnées dans cet extrait, que l'on pourra évoquer dans le même esprit comme introduction à des illustrations:

1) rapidité de transmission de l'information et rapidité de la réalisation des transactions;

2) nécessité de l'ordinateur pour la modélisation de certains instruments et de certaines stratégies;

3) sophistication accrue des acteurs qui permet la complexification des opérations.

\section{Rapidité de transmission de l'information et rapidité de la réalisation des transactions}

Des agences de presse généralistes, telle que Reuters, ou spécialisées dans le domaine de la finance, telles que Knight Ridder, Telerate, Bloomberg, ont installé des réseaux d'information immédiate qui combinent les nouvelles d'ordre économique ou d'intérêt général et l'information proprement financière comme les prix constatés et les cotations ${ }^{3}$ d'un très grand nombre de produits financiers affichés en temps réel (plus de 52000 en 1985 sur Reuters selon Mayer 1988 : 22). La première conséquence de ces innovations techniques fut la globalisation des marchés déjà mentionnée.

Une autre conséquence fut qu'une activité classique des traders, l'arbitrage stricto sensu, consistant à acheter à faible coût un produit sur un marché et à le revendre plus cher sur une autre place où il est également traité, est devenue quasiment impossible. Il était autrefois de la compétence des banques ayant de nombreuses connexions sur les places financières de repérer, par le truchement d'un réseau de correspondants téléphoniques, de telles opportunités d'arbitrage et d'en faire une source régulière de profit. L'affichage presque instantané des prix sur écrans interdit aujourd'hui la pratique de l'arbitrage stricto sensu puisque le temps, pourtant très court, entre l'achat sur un marché et la revente sur un autre, dépasse celui nécessaire au réalignement des prix sur les deux marchés, ce qui rend bien entendu l'arbitrage sans objet ${ }^{4}$. Ajoutons que de nombreuses opportunités apparentes d'arbitrage sont de simples artefacts dus au décalage existant, dans le délai d'affichage sur les écrans, entre systèmes informatiques présentant des temps de réponse différents (ainsi l'affichage du MATIF: Marché à Terme International 
de France, à Paris, est plus rapide que celui du LIFFE à Londres, bien qu'ils traitent des instruments financiers similaires) - sans compter les pseudo-opportunités d'arbitrages dues simplement aux pannes informatiques des systèmes d'information ${ }^{5}$.

L'existence d'écrans avec affichage quasi instantané des cotations et des prix des transactions effectives implique que quand on parle aujourd'hui d'arbitrage, il ne s'agit pas d'arbitrage stricto sensu mais d'opérations qui résultent d'estimations indirectes faisant apparaître que tel produit est sous-évalué ou surévalué par rapport à tel autre. Ces estimations supposent bien entendu une modélisation des équivalences postulées entre produits financiers distincts, la faiblesse éventuelle du montage résidant alors dans le niveau d'adéquation de la modélisation. Dans la pratique, ces équivalences sont postulées à partir de co-variations observées empiriquement entre données historiques relatives aux marchés concernés, c'est-à-dire à partir de l'observation selon laquelle leurs prix évoluent simultanément et dans la même direction. Rien ne garantit toutefois que les variations de prix qui auront lieu à l'avenir répéteront les co-variations qui existèrent dans le passé. En conséquence, les traders enregistrent souvent des pertes cuisantes (ce fut le cas au cours de la période février-mai 1994), lorsque des équivalences postulées, fondées sur des co-variations fortes et habituelles entre produits, se trouvent soudain démenties par les faits.

\section{Nécessité de l'ordinateur pour la modélisation de certains instruments et de certaines stratégies}

L'ordinateur permet aujourd'hui de coter, de calculer un prix à l'offre (ask) ou à la demande (bid) pour un produit dont les facteurs qui le déterminent sont trop nombreux ou la récursivité du calcul trop complexe pour que l'on puisse en imaginer une évaluation intuitive immédiate.

Par exemple, l'IRR (Internal Rate of Return), le taux actuariel qui mesure le taux de rendement effectif d'une obligation dans le contexte des marchés, ne peut s'exprimer sous la forme d'un système d'équations solubles et doit être résolu par approximations successives. Seule une calculatrice sophistiquée ou un ordinateur permettent d'en déterminer une valeur approchée en un temps raisonnable. Un calcul devant lequel la plupart des opérateurs auraient reculé il y a vingt ans est aujourd'hui effectué par l'ordinateur en quelques fractions de secondes, et l'évaluation du rendement comparé d'une multitude d'obligations acquises au comptant ou à terme peut donc être faite dans l'instant. Il faut se souvenir que l'une des raisons pour lesquelles l'ordinateur avait été conçu à l'origine était précisément de déterminer ce type de valeurs approchées ${ }^{6}$.

Une activité importante pour la gestion des opérations financières est la couverture de risque (ou hedging). Si une position sur les marchés apparaît risquée, c'est-à-dire susceptible d'entraîner des pertes considérables, il est (en principe) toujours possible de se positionner sur un autre marché dont les variations sont simultanées et ont lieu en sens opposé (l'inverse de la co-variation mentionnée ci-dessus) ou de se positionner sur le même marché mais sur un autre produit lié à ce marché et en direction opposée: acheteur au lieu de vendeur, ou inversement.

Un instrument classique de couverture est l'option ${ }^{7}$. Lacheteur d'une option sur un certain produit financier acquiert, en échange du versement d'une prime, la possibilité d'acheter (call) à un prix déterminé d'avance, prix d'exercice ou strike, une quantité 
donnée de ce produit. C'est-à-dire qu'il a le droit de profiter d'une hausse éventuelle de son prix, et cela soit un jour précis (option européenne), soit à n'importe quel moment (option américaine) ou inversement, de vendre (put) ou non à un prix déterminé d'avance une quantité donnée de ce produit, à savoir le droit de profiter d'une baisse éventuelle de son prix. Le vendeur d'une option, se trouve dans la position inverse: il perd à la hausse s'il vend un call, il perd à la baisse s'il vend un put. Pour payer une prime d'un montant moins élevé, l'acheteur d'une option peut choisir comme prix d'exercice, comme strike, un cours éloigné du cours du jour (plus élevé pour un call, plus bas pour un put); c'est là une stratégie équivalente à celle qui consiste à payer une prime d'assurance réduite en ne couvrant la perte qu'au-delà d'un montant forfaitaire.

La nature des opérations de couverture s'est modifiée du tout au tout depuis 1973, date à laquelle Fischer Black et Myron Scholes proposèrent, sous la forme d'une équation différentielle, un algorithme " objectif » de cotation (calcul d'un prix raisonnable à la vente et à l'achat d'un instrument financier) pour tous les instruments "dérivés », c'est-à-dire pour les instruments financiers dont le prix dépend de celui d'un autre instrument, classique celui-là - comme une obligation ou un titre de société - appelé son sous-jacent. Le modèle de Black et Scholes vaut en particulier pour les options dites européennes; il implique entre autres, le calcul de valeurs cumulées d'une distribution normale (gaussienne), opération que seul un ordinateur peut effectuer en peu de temps.

Bien entendu, la faisabilité mathématique de la cotation à partir de modèles complexes ne signifie pas pour autant qu'elle débouche sur des chiffres exacts, ni même que ces modèles soient conceptuellement justes. C'est le cas en particulier de la fonction différentielle proposée par Black et Scholes. Si leur équation retient comme variables quelques paramètres qui doivent nécessairement intervenir dans la formation du prix, tels que le prix actuel du produit sous-jacent, le temps qui reste à courir jusqu'à l'échéance, la volatilité (vitesse de variation du prix) et le taux d'intérêt « sans risque » (garantie d'état), elle suppose toutefois dans son expression que les marchés se comportent autrement qu'ils ne le font, par exemple, que les variations de prix suivent une loi « log normale » ou que le taux d'intérêt «sans risque » soit constant pendant toute la durée de vie de l'option et identique pour toutes les maturités.

Un danger de ce type n'est pas propre à l'informatique, il est présent dès qu'existe une formalisation mathématique: « le prix de l'option doit être exact puisqu'il résulte de la formule de Black et Scholes », n'est après tout que l'extension parmi des gens instruits du principe: «C'est vrai puisque c'est dans le journal ». L'informatisation semble cependant renforcer encore une disposition, sympathique chez l'être humain, à faire confiance, mais dont l'excès a pour nom crédulité.

\section{Sophistication accrue des acteurs permettant la complexification des opérations}

Lorsque les nouvelles sont rares et que la formation des traders est uniforme, l'ensemble des acteurs présents sur les marchés disposent d'informations identiques et évaluent de manières similaires le prix équitable pour une marchandise donnée. Dans ces conditions, les prix peuvent rester fixés pendant de longues durées à un même niveau et ne connaître que de faibles oscillations autour de celui-ci (c'est cet effet qui suggéra à Thomas d'Aquin 
la notion d'un « prix d'équilibre », notion à laquelle les économistes ont tenté de donner un fondement mathématique à partir de la fin du xix siècle).

La logique qui sous-tend « l'analyse fondamentale », pratiquée par les analystes des marchés pour déterminer des évolutions objectives des prix, considère que tout prix s'explique par l'action conjuguée d'un certain nombre de facteurs spécifiques agissant de manière connue les uns par rapport aux autres. Dans cette perspective, plus aisé est l'accès à l'information sur ces différents facteurs, plus il est loisible à quiconque de focaliser son attention sur tel aspect de la conjoncture économique pour y trouver les fondements objectifs du «juste prix » à un moment précis et pour supposer dès lors que le marché va connaître une « correction » à la hausse si le prix est sous-évalué par rapport à ce « juste prix » et à la baisse s'il est au contraire sur-évalué.

Dans une perspective similaire, plus la formation des traders est diversifiée, plus les modes de raisonnement sont sophistiqués, plus les conceptualisations du mécanisme du marché vont varier et encourager au même moment à considérer, les uns que le prix est indubitablement à la hausse, et d'autres qu'il est manifestement à la baisse. Ces conceptualisations se retrouveront sous-jacentes aux systèmes de trading « automatiques » informatisés, même si celle du mécanisme du marché résulte ici d'innombrables simulations du système et d'optimisations de paramètres à partir d'historiques de prix de marché, et semble émerger « expérimentalement » d’une objectivité « physique » de l'évolution des prix.

[...Suivait dans l'article originellement publié, une longue analyse d'une séance du marché du MATIF, marché à terme français des instruments financiers. Deux illustrations sont encore offertes: la première, le krach du marché boursier d'octobre 1987, la seconde, celle du krach du marché obligataire en 1994, contemporaine de la rédaction de l'article. La seconde met en scène les mortgage-backed securities, les titres adossés à des crédits hypothécaires, responsables déjà, quatorze ans avant la crise des subprimes d'une crise profonde des marchés financiers...]

\section{Deux illustrations complémentaires: le krach boursier de 1987 et le krach obligataire de 1994}

Les illustrations complémentaires seront traitées beaucoup plus brièvement et pour conforter simplement ce qui vient d'être exposé de manière détaillée. Elles sont empruntées toutes deux à des crises financières: le krach boursier d'octobre 1987 qui concerne au premier chef le marché des titres de sociétés ( actions ») mais aussi, comme on le verra, les futures et les options évoqués précédemment; et le krach « rampant » du printemps 1994 sur le marché des obligations. Comme nous l'avions fait observer dans un texte antérieur (Jorion 1990), les situations de crise économique ne sont pas monstrueuses au sein des contextes où elles apparaissent, elles exacerbent sans doute des traits excessifs de circonstances habituelles, mais elles présentent ainsi l'avantage de faire apparaître en surface une vérité relative aux mécanismes à l’œuvre, vérité qui demeurerait sans cela enfouie dans le foisonnement inextricable des facteurs agissants et dont les effets se neutralisent pour une large part au sein de situations dites normales ${ }^{8}$. 


\section{Le krach boursier d'octobre 1987}

Le krach d'octobre 1987 s'est esquissé le vendredi 16 par une baisse importante du marché boursier américain. Il s'est ensuite confirmé de manière brutale les lundi 19 et mardi 20 octobre. Au matin du mercredi 21 octobre 1987, 20 \% du capital des sociétés américaines s'était évanoui en fumée. Dans les jours qui suivirent, l'ordinateur se vit désigner par la presse non financière comme le principal responsable du krach. En 1987, le computer était encore essentiellement le mainframe: le « macro-ordinateur », dont l'IBM 650 fut longtemps l'exemple le plus connu.

Le marché des options eut un rôle capital dans la crise, en raison de l'engorgement dû à l'introduction à la cotation de nouveaux « prix d'exercice » (strikes) toujours moins élevés. Si l'ordinateur joua un rôle ici, ce fut probablement un rôle positif puisque les problèmes furent sans doute résolus en moins de temps qu'il n'en aurait fallu antérieurement à l'informatisation (Mayer 1988: 73).

Un autre rôle important peut être attribué aux appels de marge sur les marchés de futures de l'indice boursier Standard \& Poor 500'. Les chambres de compensation interrompirent la cotation à de nombreuses reprises pour réclamer des détenteurs de positions long (qui pariaient sur une hausse du marché et étaient donc massivement perdants) le provisionnement de leurs comptes à concurrence des sommes qu'ils étaient susceptibles de perdre lorsqu'ils sortiraient du marché; mais les chambres, elles, ne remettaient leurs marges positives à disposition des positions short (dont les profits potentiels ne cessaient de gonfler) qu'à l'échéance habituelle de la journée. Cet effet asymétrique interdit en particulier aux vendeurs qui se trouvaient en position gagnante d'utiliser leurs gains pour acheter des contrats longs, au cas où ils l'auraient souhaité, et pour freiner ainsi la chute du future Standard \& Poor 500. Dans ce cas comme dans le premier, l'informatique reste étrangère au problème.

Mais l'informatisation est sans aucun doute impliquée dans l'accélération de la chute des prix due au program trading. On entend par là une mise en œuvre conjointe, à l'aide de moyens informatiques, de la couverture dynamique (dynamic hedging) ou stratégie d'assurance du portefeuille (portfolio insurance strategy) et de l'arbitrage de l'indice boursier. Le dynamic hedging consiste à couvrir une perte éventuelle sur un portefeuille d'actions boursières en se plaçant short sur le contrat future de l'indice boursier, ce qui revient à parier parallèlement sur la baisse globale du marché. Une couverture équivalente s'obtient en achetant des puts sur l'indice ou sur les titres individuels, les puts étant, rappelons-le, des options qui s'apprécient quand le prix baisse ${ }^{10}$. Ayant calculé le bêta d'un titre, qui est une mesure de sa disposition à se comporter comme le marché boursier dans son ensemble - tel que l'indice boursier le représente -, on peut évaluer le nombre de contrats futures nécessaires pour couvrir l'ensemble de son portefeuille.

Le 20 octobre 1987, voici ce qui arriva: un si grand nombre d'institutions s'étaient assurées contre la dévaluation de leur portefeuille par la mise en place de systèmes de program trading, qu'il « cessa d'y avoir un prix » pour le contrat future Standard E Poor 500, faute d'acheteurs potentiels, et le même phénomène eut lieu sur le marché des options. Du coup, des ordres de vente «à n'importe quel prix » furent placés sur le marché, qui rencontrèrent quelques rares offres d'achat à des prix de plus en plus bas. On notera que l'effondrement des prix qui en résulta ne pourrait être expliqué dans les termes d'une théorisation où le prix refléterait une valeur; il s'explique au contraire aisément en termes de rapport de force entre vendeurs nombreux et en concurrence 
vive, et acheteurs rares, à même dès lors de fixer le prix comme bon leur semble. Mayer mentionne à ce propos le fait suivant:

«À 11 h 53 le mardi 20 octobre 1987, alors que l'indice Standard \& Poor 500 (l'indice constaté, c'est-à-dire, si l'on veut, « au comptant ») valait 218 , un contrat put novembre au niveau 195 fut vendu pour une prime de 85 - ce qui veut dire que pour obtenir le droit de vendre quatre semaines plus tard au prix de $\$ 19500$ une marchandise qui valait au même moment $\$ 21$ 800, l'acheteur était contraint de payer $\$ 8500$ » (Mayer 1988: 73).

En principe un écart trop important entre l'indice boursier (au comptant) et le prix du future de l'indice aurait dû permettre un arbitrage amortissant la chute du marché, puisqu'il était possible d'acheter le future et de vendre un «panier » de titres correspondant à la composition de l'indice. Mais cet arbitrage impliquait que l'on puisse vendre des actions, donc l'existence d'acheteurs, devenue alors plus que problématique. Par ailleurs, le système automatique qui permettait de vendre instantanément de tels « paniers » de cinq cents types différents de titres à la fois, le DOT (Designated Order Turnaround) tomba en panne.

Lorsque l'on incrimine l'ordinateur dans le krach de 1987, c'est généralement à partir d'une interprétation naïve de ce que représente l'informatique, celle où un gigantesque « cerveau électronique », semblable à ceux qui peuplaient les films de science-fiction des années cinquante, viendrait imposer sa loi à des êtres humains à la fois asservis et subjugués. Ce n'est pas de l'ordinateur sous cette forme dont il s'agit ici bien sûr, mais du rôle que l'informatisation de la finance a pu jouer dans la crise d'octobre 1987. De nombreux auteurs se sont efforcés de minimiser ce rôle, comme s'il y avait là un abominable secret que le monde de la finance se devrait de cacher aux yeux du reste du monde. Darrell Duffie fit le point sur la question sur le mode purement objectif qu'elle requiert:

«Sur le nombre record de 162000 contrats futures Standard \& Poor 500 qui furent traités le 19 octobre, selon la Commodity Futures Trading Commission, la stratégie d'assurance de portefeuille rend compte de 12 à $24 \%$ des transactions. L'estimation relative au 20 octobre est de 19 à $26 \%$. (Ce sont là les chiffres qui furent publiés par le New York Times). Larbitrage de l'indice ne représenta que $9 \%$ du trading le 19 et moins de $2 \%$ le 20, toujours selon la même source » (Duffie 1989 : 161).

\section{Le krach sur les marchés obligataires (février-mai 1994)}

Nous avons déjà fait allusion à plusieurs reprises à ce krach larvé qui intervint durant la période de rédaction du présent article et qui permit dès lors une sorte d'illustration en direct des hypothèses que nous voulions tester. Plusieurs facteurs ont joué lors de cette crise, mais le principal a sans doute été la répercussion, sur le marché financier dans son ensemble, des difficultés rencontrées par le marché des mortgage-backed securities aux États-Unis. Celles-ci sont des titres constitués en rassemblant en portefeuilles des hypothèques contractées à titre individuel par des particuliers. Un grand nombre de possibilités existent quant à la manière de créer ainsi des titres aux caractéristiques spéciales: par exemple, et pour citer les cas les plus simples des stripped mortgage-backed securities, des titres constitués uniquement du paiement des intérêts par les particuliers, et d'autres constitués du seul remboursement amorti du principal. En gros, le mécanisme de la crise a été le suivant, si l'on ne retient que le rôle joué par les mortgage-backed securities. Comme on l'a dit plus haut, pour couper court à un risque d'inflation dû à une 
surchauffe de l'économie américaine, la Federal Reserve Bank releva à plusieurs reprises au cours du printemps 1994 les taux court terme. Dans toutes ces occasions, le marché des mortgage-backed securities connut un nouveau soubresaut, et une pareille accumulation conduisit en avril à une faillite retentissante, celle de Askin Capital Managements (Granite Capital et Granite Partners) dont le propriétaire était David Askin, grand détenteur de tels titres. Selon un principe tout à fait général, un particulier peut refinancer son prêt au logement à mesure que les taux baissent, en remboursant son emprunt actuel grâce à un nouvel emprunt à un taux moins élevé. Une telle possibilité de refinancement rend ces titres d'un type particulier que sont les mortgage-backed securities très sensibles aux variations des taux de marché. Une augmentation des taux d'intérêt rend au contraire les contractants d'hypothèques moins aptes à rembourser leur dette, et par conséquent, elle allonge la vie moyenne (notion de duration) d'un portefeuille de titres du type des mortgage-backed securities.

Une hypothèque est une dette et sa logique sous-jacente s'apparente donc à celle qui sous-tend toute dette, et en particulier les obligations émises par les États ou les emprunts obligataires émis par les entreprises. Plus la maturité d'une obligation est lointaine, plus elle est exposée au risque de défaut de la contrepartie (non-remboursement de la dette), et par conséquent, plus elle est risquée. Pour réduire le risque de leur portefeuille global, les détenteurs de mortgage-backed securities tentèrent donc de réduire sa durée de vie en vendant des obligations de longue durée qu'ils détenaient par ailleurs. Un exemple typique de ces obligations est la Treasury Note du Trésor américain dont la maturité est de 10 ans, un autre exemple en étant le Treasury Bond dont la maturité est de 20 ans (Wessel, Jereski et Smith 1994). À chacun des relèvements des taux courts par la Réserve Fédérale, de grandes quantités de Treasury Notes et de Treasury Bonds (ou de contrats short sur le future du Treasury Bond) se retrouvèrent offertes simultanément sur le marché, avec pour conséquence une baisse brutale de leur prix (Taylor 1994).

Voici maintenant le mécanisme sous-jacent à ce qui devint le krach obligataire de 1994. Une obligation a un coupon qui lui est attaché. La valeur de l'obligation reflète la valeur comparative de ce coupon par rapport au taux d'intérêt qu'offre, au même moment, le marché. Si une obligation coûte $1000 \mathrm{~F}$ au moment de son émission avec un coupon annuel de, par exemple, $8 \%$, elle vaudra moins de $1000 \mathrm{~F}$ le jour où le marché proposera un coupon annuel de $8,5 \%$ - une obligation qui offre ce rendement plus élevé étant nécessairement plus attractive; inversement, si le marché n'offre plus que $7 \%$, le prix de revente d'une obligation à coupon $8 \%$ dépassera cette fois les $1000 \mathrm{~F}$. Par une lecture inversée, une obligation dont le prix baisse est automatiquement une obligation qui reflète un relèvement du taux de marché correspondant à sa maturité.

En conséquence, plus les obligations se dépréciaient sur le marché du fait de la présence de vendeurs nombreux et d'acheteurs rares, plus le taux d'intérêt implicite à ces obligations croissait, établissant sur le marché un nouvel étalon pour des emprunts de cette maturité, mouvement des taux auquel les ménages, les entreprises et l'économie dans sa totalité se trouvaient alors nécessairement soumis. Comme nous l'avons signalé, le prix des obligations françaises participa au mouvement général consécutif aux mesures prises la Federal Reserve.

$$
[\ldots]
$$

Le fait de drainer aujourd'hui les meilleurs esprits vers les activités financières contribue sans doute à favoriser la rapidité des effets de rétroaction dans ce secteur. La peur de perdre des sommes qui se chiffrent en millions sinon en milliards concourt sans aucun doute au feed-back négatif, celui qui ramène vers la stabilité, mais la confiance en soi qui 
accompagne une qualification toujours plus poussée encourage les effets de feed-back que le cybernéticien appelle à l'inverse, positif, ceux qui se confondent avec les phénomènes « d'emballement ${ }^{11}$. Seule la disposition typiquement humaine à l'hésitation vient ordinairement tempérer cette tendance. L'ordinateur qui a été programmé pour prendre les mêmes décisions lorsque les circonstances sont identiques vient, lui, renforcer la disposition à l'emballement. Paradoxe de la nature humaine, le comportement pourtant froid et objectif de l'ordinateur se voit alors attribuer du sens, comme s'il résultait de l'intentionnalité d'un autre acteur humain.

Lorsqu'a été mis en évidence, au long des pages qui précèdent, le rôle de l'informatisation dans la crise financière de 1987 ou dans celle de 1994, il ne s'agissait nullement du « cerveau électronique-maître du monde », mais d'une micro-informatique entièrement maîtrisée, dans sa conception comme dans son fonctionnement, par les êtres humains qui y ont recours. Que ces êtres humains soient sujets à l'erreur est évident, mais les crises évoquées ici ne résultent ni d'erreurs ni même de maladresses dans l'utilisation de l'outil informatique: elles découlent essentiellement de la puissance intrinsèque qui est la sienne et des interférences produites entre les implications diverses de la technique et la propension humaine à se prendre au jeu d'intentions qu'il s'agit de deviner. En faisant « monter au créneau » un être dont la logique est celle du seul calcul, l'informatisation de la finance lui a permis de faire agir en son nom un agent sensible à la seule logique d'une Raison absolue, imperméable aux faiblesses de l'émotion humaine et réceptacle impuissant de tout fantasme d'intentionnalité, c'est-à-dire de responsabilité, dans les crises dont il est effectivement devenu le catalyseur.

Le mécanisme de la crise obligataire de 1994 qui se déroula « en direct » pendant que nous écrivions le présent article, est révélateur de l'influence majeure qu'exerce aujourd'hui l'informatique sur la finance et de l'influence majeure qu'exerce en contrecoup la finance sur l'économie de production. Si M. Askin pouvait supposer avoir mis au point des portefeuilles d'hypothèques regroupées qui ne se dépréciaient en aucun cas (ses collègues s'accordaient à lui reconnaître ce talent), c'est certainement à partir d'études extrêmement poussées quant aux possibilités de couverture de ces portefeuilles, c'est-à-dire à partir d'analyses statistiques en termes de degré de co-variation de différents marchés, qui exigent une capacité de traitement des données dont seul l'ordinateur dispose. Dans un cas comme celui-ci, le gigantisme des calculs communique aux chiffres générés une « objectivité » qui fait croire à leur permanence. Une telle naïveté ne date pas d'aujourd'hui; elle accompagne depuis toujours le recours à l'outil mathématique mais l'informatique vient la renforcer à partir de l'idée que «l'ordinateur ne se trompe pas».

Le fait que l'ordinateur ne se trompe pas « algorithmiquement » n'empêche pas le modèle à partir duquel il opère d'être éventuellement faux, et les modèles de la théorie financière le sont certainement puisqu'ils n'intègrent pas - jusqu'à présent du moins - la rétroaction qui résulte de la modification du comportement des acteurs au vu des variations de prix. Dans les modèles financiers existants - c'est le cas en particulier du modèle Black-Scholes de cotations des options mentionné plus haut -, les effets de la rétroaction sont agrégés et redéfinis comme constituant collectivement un phénomène stochastique, autrement dit, un phénomène se produisant « au hasard ». L'analogie sous-jacente est celle de la mécanique statistique; l'erreur réside bien entendu dans le fait que le comportement réfléchi, « orienté », des opérateurs de marché est assimilé à l'entrechoquement « insignifiant » de particules. Le plus paradoxal sans doute est que ce sont les mêmes acteurs qui considèrent d'une part que «le prix est une information », et d'autre part que «les 
variations de prix sont aléatoires »: ou bien en effet, le prix constitue une information (interprétable), et dans ce cas les décisions qui en découlent, et qui vont contribuer à modifier ce prix, n'engendrent certainement pas des variations aléatoires, ou bien les interventions décidées à partir de l'interprétation du prix conduisent effectivement à des variations aléatoires de celui-ci et dans ce cas, la représentation selon laquelle le prix constitue une « information » est illusoire. La réalité, comme nous l'avons vu, est celle-ci: les variations de prix constituent quelquefois une information et quelquefois non; quoi qu'il en soit, elles sont interprétées a priori comme étant significatives et débouchent sur des comportements qui se manifestent à leur tour en tant que variations de prix (qui constitueront quelquefois une information et quelquefois non).

Le fait que les obligations se vendent et s'achètent aujourd'hui au comptant et à terme, dans des conditions de rétroaction rapide qui encouragent les variations amples de leur prix, signifie que les taux d'intérêt qui sont inscrits dans ces prix varient dans la même proportion.

Or ces taux ne sont pas fiduciaires, cantonnés à leur seule logique d'instruments financiers: ce sont les mêmes taux qui président aux mouvements de capitaux sur lesquels repose l'économie de la production tout entière: prêts aux entreprises et prêts aux ménages. L'ironie de la crise obligataire du printemps 1994 - si l'on s'en tient au rôle joué par les seules mortgage-backed securities-, c'est qu'une confiance excessive accordée à des simulations portant sur le rendement des portefeuilles de prêts au logement, contribua à la hausse brutale des taux d'intérêt, c'est-à-dire, barra de fait l'accès aux prêts au logement pour un nombre considérable de candidats et cela à l'échelle de la planète entière. La queue de la finance remue désormais le chien de l'économie, jusqu'à lui en donner le tournis.

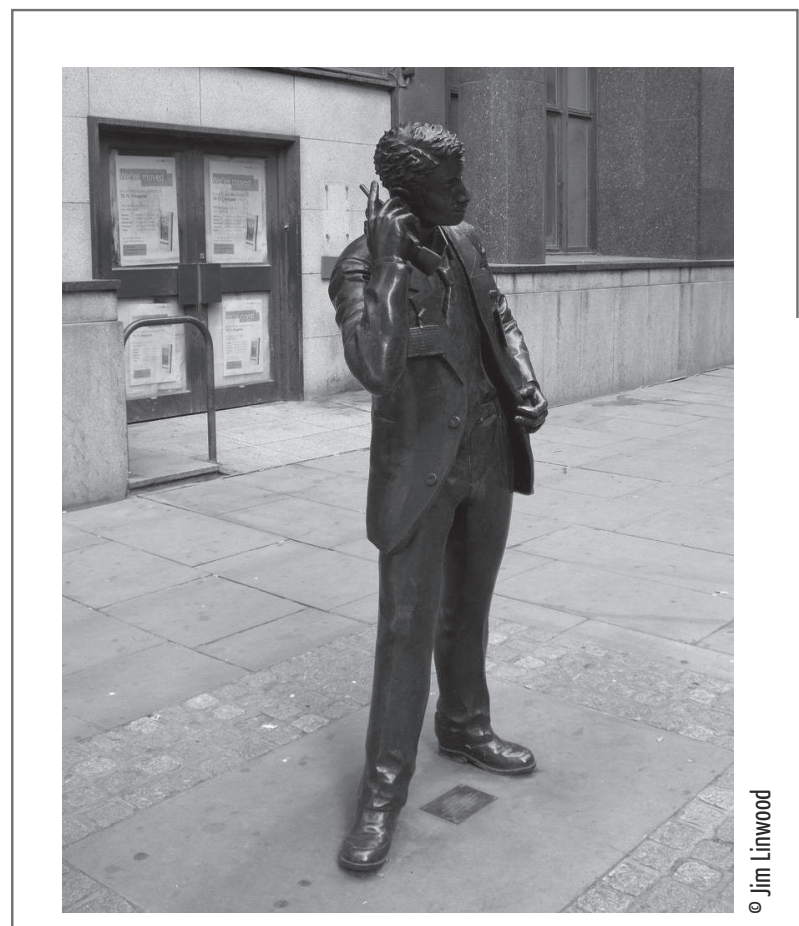

La statue du « trader », Londres

Une statue des plus insolites sur Walbrook Street, un trader en plein milieu de la rue. 
1. Voici un exemple, extrait d'un article publié dans la presse: «II n'est pas surprenant que certaines des figures de proue dans le domaine des instruments dérivés n'ont pas acquis leur formation dans les 'business schools' mais dans les écoles d'ingénieurs telles le Massachusetts Institute of Technology. Charles Dexter de la Chase Manhattan Bank y a acquis ses diplômes en aérospatiale et en finance» (Hiltzik 1994). Et un autre: «Ce n'est plus un secret que les physiciens d'orientation mathématique trouvent un environnement favorable dans la banque et les autres secteurs de la haute finance en tant qu'analystes quantitatifs. 'II y a des tas de types dans mon cas ici', résume Albert Zisook de la Swiss Bank Corporation» (Robinson 1994: 55)

2. Nous avions hésité devant une définition qui pouvait sembler sujette à controverse, quand nous sommes tombés dans un ouvrage récent sur le passage suivant: 'Du fait que la valeur de ces bouts de papier est toujours davantage fonction du prix d'autres bouts de papier, et non d'une production et d'une consommation réelles, une nouvelle culture financière apparaît, qui se nourrit d'elle-même'. (Mayer 1993 : 45).

3. On distingue les prix effectivement constatés lors de transactions réelles, des cotations, qui sont des prix demandés par des acheteurs potentiels (bid) ou offerts par des vendeurs potentiels (ask) et qui demeurent «virtuels» aussi longtemps que des transactions réelles n'ont pas eu lieu à leurs niveaux.

4. Sur les marchés authentiquement symétriques comme les marchés de futures [... ou l'on peut se placer indifféremment comme «vendeur» (position dite short) ou comme «acheteur» (position dite long), l'ordre des opérations est lui aussi indifférent et l'on peut, en principe, arbitrer en vendant d'abord et en achetant ensuite.

5. Ainsi, comme le note un commentateur du krach de 1987, «le 19 octobre, le système de trading du New York Stock Exchange avait un retard cumulé sur les ordres non traités qui atteignait quarante-cinq minutes. Bien entendu, si les prix de futures d'indices boursiers étaient remis à jour sur les écrans plus rapidement que les prix des titres, on pouvait avoir l'impression qu'il existait des opportunités d'arbitrage là où en réalité il n'y en avait pas.» (Duffie 1989: 160). Notons qu'a fortiori lorsqu'un arbitrage apparaît possible sur des chiffres publiés dans un journal, il y a bien longtemps que la possibilité d'arbitrer effectivement s'est évanouie (voir Hull 1993 : 142).

6. En 1937 Howard Aiken, instructeur au Département de Physique de l'Université de Harvard, fut confronté dans les recherches connexes à sa thèse, à des équations différentielles qui ne pouvaient être résolues analytiquement et dont la solution numérique impliquait une somme de travail considérable; il entreprit de concevoir une machine à calculer automatique (Randell 1973 : 191). C'est ainsi que naquit Mark l, le premier ordinateur produit par IBM en 1943.

7. Puisque le prix d'une option est lié à celui du produit, les options peuvent être utilisées en couverture. (Brealey et Myers 1991 : 643).

8. Nous écrivions à propos du contingentement à la pêche : «Pourquoi [ ... ] ne pas postuler une hypothèse inverse de celle prônée par la science économique, à savoir que la concertation dans (le contingentement) ne constitue pas un cas aberrant, simple infraction aux règles, mais constitue un cas de lisibilité en surface d'un mécanisme qui demeure sinon caché ; autrement dit, une traduction de l'implicite en explicite rendue nécessaire par des circonstances difficiles $7 »$. (Jorion 1990 : 95)

9. Les deux principaux indices Standard \& Poor, sont le S\&P 100, calculé sur cent titres, et le S\&P 500, calculé sur cinq-cents. Le contrat future le plus populaire est celui qui porte sur le S\&P 500. L'option sur le S\&P 100 (appelée OEX) est de type américain (i.e. peut être exercée à tout moment), l'option sur le S\&P 500 (appelée SPX) est de type européen (i.e. ne peut être exercée qu'à l'échéance du contrat). C'est l'OEX qui joua un rôle majeur lors du krach de 1987 (Daigler 1994 : 322-323)

10. Précisons à l'intention des puristes que le prix d'exercice ou strike des puts doit alors être le prix actuel du marché, faute de quoi les deux stratégies ne sont pas strictement équivalentes.

11. Nous écrivions dans un précédent texte: «L'éventualité du krach financier est liée à cette possibilité d'emballement dans une seule direction que l'on appelle alors feedback positif. Une barque qui chavire parce que ses oscillations conduisent ses passagers à se porter trop rapidement d'un bord sur l'autre pour tenter de contrebalancer une gîte qui s'accentue, présente le même type de phénomène.» (Jorion 1994 : 176). 


\section{RÉFÉRENCES}

Brealey, R.A. \& S.C. Myers 1991 Principles of corporate finance. New York: McGraw-Hill (4th ed.).

Bruce, R. 1994 When the tail wags the market dog, Independent on Sunday, 20th February.

Daigler, R.T. 1994 Financial futures and options markets. Concepts and Strategies. New York: Harper Collins.

La Torre, J. (de) 1994 International finance has become the Dog-Wagging Tail of the '90s, Los Angeles Times, 20th March.

Duffie, D. 1989 Future markets. Englewood Cliffs (NI): Prentice Hall.

Fabozzi, FI., F. Modigliani \& M. G. Ferri 1994 Foundations of financial markets and institutions. Englewood Cliffs (NI) : Prentice-Hall International.

Hansell, S. 1994 Costly lesson for Wall Street whiz. Askin ans investors find there's no perfect hedge, International Herald Tribune, 6th April.

Hiltzik, M.A. 1994 Derivatives: perils of ingenuity, Los Angeles Times, 12th May.

Hull, J. 1993 Options, futures, and other derivative securities. Englewood Cliffs (N.J.): Prentice-Hall International ( $2 \mathrm{~d}$ ed.).

Jorion, P. 1990 Déterminants sociaux de la formation des prix de marché. L'exemple de la pêche artisanale, La Revue du MAUSS semestrielle 9: 71-106; $10: 49-64$.

— 1994 L'Économique comme science de l'interaction humaine vue sous l'angle du prix. Vers une physique sociale, in Pour une autre économie, revue du MAUSS semestrielle: 161-181.

Kansas, D. 1994 Squeeze in U.S. May soon turn short sellers' jubilation into Gloom, Wall Street Journal Europe, 22d August.

Mayer, M. 1988 Markets. New York: Norton.

— 1993 Nightmare on Wall Street. Salomon Brothers and the Corruption of the Marketplace. New York: Simon \& Schuster.

Natenberg, S. 1994 Option volatility and pricing, advanced trading strategies and techniques. Chicago: Probus.

Randell, B. (ed.) 1973 The Origins of digital computers. Berlin: Springer-Verlag.

Robinson, A.L. 1994 The Physics of high finance, Physics Today, June: 55-56.

Schwager, J.-D. 1992 The New market wizzards. Conversations with america's top traders. New York: HarperBusiness.

Skidelsky, R. 1992 John Maynard Keynes, volume two, the economist as saviour, 1920-1937. London : MacMillan.

Sobel, R. 1993 Dangerous dreamers. The financial innovators from Charles Merrill to Michael Milken. New York : John Wiley.

Taylor, J. 1994 Bond volatility adds to frenzy of futures pits, Wall Street Journal Europe, 18th May.

Wessel, D. 1994 L. Jereski, R.Smith, Stormy spring. Three-month tumult in bonds lay bare new financial forces, Wall Street Journal Europe, 25th May. 


\section{RÉSUMÉ}

La queue qui remue le chien. Métamorphose de la finance due à son informatisation. La finance a connu une évolution rapide au cours des quinze années qui nous séparent de la naissance de l'ordinateur individuel. L'accélération dans la diffusion de l'information, la rapidité des transactions et la possibilité de calculer des prix au moyen de formules complexes permettent à l'être humain de mettre en avant une machine qui ignore la peur devant la perte, émotion qui amortissait jusqu'ici des variations de prix enclines à l'emballement à la hausse ou à la baisse. En retour, l'homme attribue au calcul effectué par l'ordinateur ou à son affichage du résultat de l'activité humaine, des significations illusoires. L'éventualité de la crise financière ou krach est favorisée par une informatique qui permet à ses utilisateurs de concevoir la finance comme fondée sur une rationalité absolue.

\section{ABSTRACT}

The Tail that wags the dog. Metarmophosis of finance due to data-processing. Finance has undergone a rapid evolution during the fifteen years that separates us from the birth of personnel computer. The acceleration in the dissemination of information, the speed of transactions, and the possibility of calculing prices by means of complex formaulas allow human beings to produce a machine unafraid of a loss, an emotion that up until now dampened price variations prone to runaway highs or lows. In return men attributed illusory meanings to the calculations made by computers or to the computers' posting of the results of human activity. The contingency of a financial crisis or crash is enhanded by data processing which leads its users to conceive of finance as based on absolute rationality.

\section{MOTS CLÉS}

Bourse, finance, informatique, krach, prix, taux d'intérêt.

\section{KEYWORDS}

Stock Market, finance, data processing, crash, price, interest rate.

\section{NOTES ET RÉFÉRENCES DE L'INTRODUCTION}

1. Vers la crise du capitalisme américain? (La Découverte 2007), réédité sous le titre La crise du capitalisme américain (Le Croquant 2009). 